\section{REVISTA Mario Alario JURIDICA D'Filippo}

\title{
Hacia la construcción de un mercado único digital de América latina: El Geo-bloqueo en Europa $^{1}$
}

\author{
Towards the construction of a single digital market in Latin America: \\ The Geo-blockade in Europe \\ Olga Mayorga Cerpa ${ }^{2}$ \\ Université de perpignan via domitia - Francia
}

\section{RESUMEN}

El Internet es indiferente a las fronteras y como red transnacional es objeto de innumerables problemas jurídicos que afectan el mercado digital y la libertad de acceder a los contenidos y servicios en línea. Uno de los proyectos de la estrategia digital 2020 de la CEPAL es la creación de un mercado único digital en América latina, en este sentido, es indispensable adaptar el sistema jurídico de los países latinoamericanos a la era de la economía digital, para ello, se realizará un trabajo de Derecho comparado de la legislación europea en materia de bloqueo geográfico de los contenidos, bienes y servicios digitales. El presente artículo tiene como objetivo impulsar el desarrollo del Derecho digital e inmaterial, procurando que la doctrina jurídica de Colombia se convierta en uno de los pioneros en contrarrestar las formas de discriminación en línea e incentivar el desarrollo de un mercado digital en América latina.

Palabras clave: Mercado único digital; Geo-bloqueo; Territorios digitales; la Economía digital.

\begin{abstract}
The Internet is indifferent to borders and as a transnational network it is the subject of innumerable legal problems that affect the digital market and the freedom to access content and services online. One of the projects of CEPAL's 2020 digital strategy is the creation of a single digital market in Latin America, in this sense, it is essential to adapt the legal system of Latin American countries to the era of the digital economy. will carry out a comparative law work on European legislation on geo-blocking of digital content, goods and services. This article aims to promote the development of digital and intangible law, ensuring that the legal doctrine of Colombia becomes one of the pioneers in countering forms of online discrimination and encouraging the development of a digital market in Latin America.
\end{abstract}

Keywords: the digital market; geo-blocking; digital territories; the digital economy.

\footnotetext{
${ }^{1}$ La presente investigación es un trabajo independiente que se deriva de la tesis de grado de maestría titulada "les enjeux juridique du geoblocage", y constituye un ejercicio de derecho comparado destinado al desarrollo del derecho digital en América latina y tomando como referencia las actuales directivas del derecho europeo.

${ }^{2}$ Abogada de la Universidad de Cartagena. Master en derecho de los negocios. Master en derecho económico de los mercados nacionales e internacionales de l’Université de Perpignan Via Domitia, olgalore93@ hotmail.com
} 


\section{INTRODUCCIÓN}

La creación de un mercado interior ha constituido uno de los pilares más importantes de la Unión Europea, el cual, fue diseñado como un espacio sin fronteras que ofrece a sus residentes una libre circulación de mercancías, de servicios, de capitales y de personas suprimiendo todas las barreras que podrían representar un obstáculo para su ejercicio. Sin embargo, el incremento masivo de las interacciones entre los consumidores al interior del mercado digital europeo y el manejo desmesurado de datos personales y no personales ha ocasionado que las empresas y los proveedores de servicios digitales utilicen técnicas que les permitan bloquear y limitar el acceso de algunos clientes, fragmentando el mercado, una de estas es conocida como el geo-bloqueo o bloqueo geográfico.

En lo referente a los países latinoamericanos, el Banco de Desarrollo de América Latina ((CAF), 2016) propuso el proyecto de estrategia para la conformación de un mercado único digital (MUD) en América Latina. A través de su informe, la CAF informó sobre las dificultades que tienen estos países para llevar a cabo este proyecto, teniendo en cuenta la precaria infraestructura de la red de telecomunicaciones, sector que fue desarrollado en la mayoría de los países de América Latina tan solo a partir de la década de 1990, además del mínimo porcentaje de desplazamientos entre la población de los países de América Latina. En este sentido, no existe un mercado digital que conecte a los países de la región y que permita una libre portabilidad transfronteriza de los contenidos en línea como en el caso del contexto institucional de la Unión Europea.

A pesar de lo anterior, la presente investigación se justifica en el incremento actual en la circulación de datos dentro de los territorios digitales y al acelerado desarrollo de los mercados digitales internacionales, lo cual, ha ocasionado que los sistemas jurídicos deban estar en la capacidad de contrarrestar las problemáticas que afectan a los consumidores en línea, entre ellas, el Geo-bloqueo. De esta manera, se tomará como referencia la legislación europea, la cual, ha sido pionera en la regulación de esta técnica tecnológica.

Inicialmente se presentará el concepto de Geo-bloqueo identificando sus principales características y los elementos que la diferencia de otro tipo de métodos de restricción acceso de los consumidores, este análisis se realizará con base al principio de neutralidad del internet en Europa, el cual, ha servido como base para la regulación jurídica de esta técnica de bloqueo. Posteriormente, será indispensable desarrollar el concepto de "justificación objetiva" para comprender cuando es permitido realizar un bloqueo geográfico sin que constituya una forma de discriminación en línea. (I) 
Posteriormente, será indispensable hacer referencia al enfoque geográfico de internet, lo cual, permite concebir la existencia real de un Derecho digital e inmaterial que traspasa el tradicional requisito de la territorialidad jurídica. De esta manera, se desarrollará el concepto de territorios digitales y de los mercados digitales tanto europeos como el proyecto de creación en América latina. Teniendo en cuenta este contexto, se expondrá la manera como el geobloqueo utiliza la localización geográfica para poder comprender los efectos que esta práctica injustificada produce en el mercado digital. (II)

Finalmente, se realizará un análisis de la reglamentación europea que prohíbe el Geo-bloqueo injustificado como forma de discriminación y obstáculo del mercado único digital, estudiando cuales son las principales modificaciones que el reglamento 2018/302 ha ocasionado dentro del mercado europeo. Igualmente, es indispensable responder al interrogante de eficacidad del reglamento por lo cual se expondrán las sanciones que hasta la fecha han sido pronunciadas por la Comisión Europea frente a la práctica injustificada de Geo-bloqueo, con el objetivo de presentar recomendaciones para su desarrollo en Colombia. (III)

\section{El concepto del geo-bloqueo y el principio de neutralidad del internet en Europa}

El acceso a Internet es considerado un derecho reconocido en virtud de la libertad de expresión y el derecho a la información por el Tribunal Europeo de Derechos Humanos y el Consejo de Derechos Humanos de la ONU, dado que su existencia responde a la importancia de adecuar la ley a las nuevas tecnologías y asegurar la indiscutible necesidad de conexión de la población. Sin embargo, "la protección de la libertad de acceso a Internet como componente de la libertad de expresión, solo se garantiza con la condición de que se garantice la neutralidad de Internet, lo que implica el libre acceso para todos a todos los contenidos de Internet". (Maire, 2019).

El Reglamento (CE) 2015/2120 del Parlamento Europeo y del Consejo de 25 de noviembre de 2015 por el que se establecen medidas relativas al acceso a un internet abierto, ha consagrado el principio de neutralidad de la net según el cual "los proveedores de servicios de acceso a internet tratarán todo el tráfico de manera equitativa cuando presten servicios de acceso a internet, sin discriminación, restricción o interferencia, e independientemente del emisor y el receptor, el contenido al que se accede o que se distribuye, las aplicaciones o servicios utilizados o prestados, o el equipo terminal empleado". (Reglamento (UE), 2015/2120).

Así mismo, como explica el profesor (Derieux, 2010) la neutralidad en la net es uno de los elementos y fundamentos del derecho al internet y "el marco regulador vigente busca fomentar la capacidad de los usuarios finales para 
acceder a la información y distribuirla o ejecutar aplicaciones y servicios de su elección" (Reglamento (UE), 2015/2120).

En este sentido, la neutralidad de Internet es un principio reconocido por el derecho europeo y "destinado a garantizar la igualdad de trato de todos los flujos de información en Internet para que no sean bloqueados, degradados o favorecidos por los operadores de telecomunicaciones. Sin este principio, los operadores de telecomunicaciones tenderían a favorecer a los usuarios de sus sitios y servicios o incluso a bloquear o ralentizar intencionalmente ciertos sitios o servicios" (Boggs, 2017). Ahora bien, este principio no es absoluto por lo que los proveedores de servicios de Internet y las grandes plataformas digitales podrían aplicar medidas razonables para gestionar el tráfico de Internet, pero deben abstenerse de "bloquear, ralentizar, alterar restringir, interferir o degradar contenidos, aplicaciones o servicios específicos, o categorías específicas de estos, ni de establecer discriminaciones entre ellos" (Reglamento (UE), 2015/2120) sin justificación.

Ahora bien, una vez que el usuario ha obtenido acceso a Internet, su derecho y libertad pueden ser atacados por diferentes tipos de discriminación. El Geobloqueo es definido por la European Commission (2016) como "una práctica para restringir el acceso de bienes de consumo en línea o el acceso a contenido digital en función de la ubicación geográfica del consumidor". Esta medida tecnológica es utilizada por los operadores de telecomunicaciones como una estrategia de fragmentación del mercado al dirigirse a determinados consumidores y usuarios y discriminar a otros por su ubicación geográfica.

De la misma manera, se practican otras técnicas para restringir el acceso de los cyberconsumidores que no deben confundirse con el Geo-bloqueo, como es el caso del "re direccionamiento automático" o "re direccionamiento" mediante el cual "el consumidor puede ser redirigido automáticamente a otro sitio web dedicado, a veces sin necesariamente ser informado, dependiendo de la ubicación de su dirección IP" (Europeo, 2013); y, paralelamente, se encuentra la denegación de venta al momento de efectuar el pago y que consiste en impedir su conclusión o la entrega de la mercancía en razón a la residencia o el domicilio bancario del consumidor.

En este sentido, se hace referencia al Geo-bloqueo cuando "los comerciantes que ejercen su actividad en un Estado miembro bloquean o limitan el acceso a sus interfaces en línea, tales como sitios web y aplicaciones, a clientes de otros Estados miembros que desean realizar transacciones transfronterizas (práctica conocida como «bloqueo geográfico»). También sucede cuando determinados comerciantes aplican a esos clientes de otros Estados miembros condiciones generales de acceso diferentes a sus productos y servicios, tanto en línea como fuera de línea" (Reglamento (UE), 2018). En consecuencia, esta técnica está dotada de una característica de extranjería de 
la relación entre las partes, por lo que el vendedor / distribuidor debe realizar su actividad en un Estado miembro de la Unión Europea diferente al Estado de origen o ubicación del consumidor lo que convierte al Geo-bloqueo en una forma de discriminación.

El Geo-bloqueo tradicionalmente ha tenido una doble connotación; por una parte, es una medida de protección tecnológica, en este sentido, el Geobloqueo se considera como justificado; en contraposición, se ha determinado como una forma de discriminación al acceso de bienes y servicios de los consumidores digitales en razón a su nacionalidad, lugar de residencia o lugar de establecimiento, siendo injustificado.

\subsection{La exigencia de justificación objetiva}

En razón al avance y desarrollo del comercio electrónico y las diversas transacciones transfronterizas que tienen lugar en el mercado de Internet, es fundamental entender que una libertad absoluta del consumidor resulta imposible de concebir, y por tanto la segmentación de los consumidores solo se permitirá sobre la base de razones objetivas que justifiquen su aplicación, en este sentido, el bloqueo geográfico será una restricción de acceso permitida y no será sancionado como práctica discriminatoria porque existe una justificación objetiva para su aplicación porque no viola el artículo 20 de la Directiva 2006/123/CE (2006), el cual, establece que:

"Los Estados miembros harán lo necesario para que las condiciones generales de acceso a un servicio que el prestador ponga a disposición del público no contengan condiciones discriminatorias basadas en la nacionalidad o el lugar de residencia del destinatario, sin que ello menoscabe la posibilidad de establecer diferencias en las condiciones de acceso directamente justificadas por criterios objetivos" (Directiva Europea, 2006)

La Comisión Europea (2016) ha reconocido que el geo-bloqueo estará justificado cuando su aplicación resulte de restricciones legales impuestas por los estados miembros y que sean independientes de la voluntad del vendedor o distribuidor de un producto o servicio.

En ese orden de ideas, el Reglamento 2018/302 (2018) prevé que "en algunos casos, el bloqueo o la limitación de acceso o la redirección sin el consentimiento expreso del cliente a una versión alternativa de una interfaz en línea por motivos relacionados con la nacionalidad, con el lugar de residencia o con el lugar de establecimiento del cliente pueden ser necesarios para garantizar el cumplimiento de un requisito legal establecido en el Derecho de la Unión, o en la legislación de un Estado miembro de conformidad con el Derecho de la Unión, al que esté sujeto el comerciante como consecuencia de que ejerce su actividad en dicho Estado miembro". Debe indicarse que, en todo caso, su prohibición no deberá afectar el ejercicio de la libertad de expresión o la libertad de prensa (Unión Europea, 2000). 
A manera de ilustración, las entidades privadas que operan en un Estado miembro pueden bloquear o limitar el acceso de clientes de otros Estados miembros cuando existan motivos que puedan justificar las diferencias en las condiciones de acceso, por ejemplo, en el caso de que las microempresas y las pequeñas y medianas empresas (PYME) que no puedan garantizar el acceso a todo el territorio digital europeo, podrán hacer uso de técnicas de geo-bloqueo que le permitan delimitar su mercado.

Otros motivos por los que el Geo-bloqueo se considera justificado son "los entornos jurídicos divergentes, la incertidumbre jurídica que ello implica, los riesgos asociados a la legislación aplicable en materia de protección de los consumidores, la legislación en materia de medio ambiente o etiquetado, las cuestiones tributarias y fiscales, los gastos de entrega o los requisitos lingüisticos” (Reglamento (UE), 2018).

\subsection{El Geo-bloqueo injustificado como forma de discriminación}

Como regla general, está prohibido en el territorio europeo realizar prácticas para "dividir artificialmente el mercado interior sobre la base de las fronteras nacionales para obstaculizar la libre circulación de bienes y servicios, limitando así los derechos de los clientes e impidiendo una elección más amplia y unas condiciones óptimas" (Reglamento (UE), 2018). En consecuencia, será sancionada toda forma de discriminación basada en la nacionalidad, lugar de residencia o lugar de establecimiento de los clientes que obstruyan el mercado único digital europeo, pero solo en situaciones en las que tal trato diferenciador no tenga una justificación objetiva.

El Geo-bloqueo injustificado incluye todas las técnicas de restricción de acceso por un criterio geográfico sin que exista una justificación objetiva, como el bloqueo del acceso transfronterizo a sitios web, productos o precios, con o sin redirección a sitios "nacionales", la autorización de acceso transfronterizo a ofertas y sitios web, también para evitar que el usuario finalice su pedido o su compra, impedir la descarga de productos digitales, la autorización de acceso transfronterizo a ofertas y sitios web pero impidiendo la recogida, entrega o envío transfronterizo de mercancías, la aplicación de diferentes condiciones o precios según la nacionalidad del cliente, el país de residencia o el lugar desde el que realiza el pedido o accede a los servicios, la denegación de acceso a servicios adicionales (tarjetas de fidelización, descuentos, cupones de descuento) y la denegación de acceso a servicios posteriores -venta (reparación y mantenimiento de un producto, atención al cliente), entre otras son formas de geo-bloqueo (Reglamento (UE), 2018).

Por lo anterior, el Consejo y el Parlamento Europeo publicaron el Reglamento 2018/302 de 28 de febrero de 2018 relativo a medidas destinadas a impedir el bloqueo geográfico injustificado y otras formas de discriminación por razón de la nacionalidad, del lugar de residencia o del lugar de establecimiento de los clientes en el mercado interior y por el que se modifican los Reglamentos (CE) no 2006/2004 y (UE) 2017/2394 y la Directiva 2009/22/CE. 


\section{Los territorios digitales y la geolocalización: dimensión geográfica del internet}

Actualmente, el concepto tradicional de territorio ha evolucionado, por lo que sus fronteras se han ido desplazando y adaptando según los cambios impuestos por el mundo digital, estos territorios se denominan virtuales y son definidos por el autor (Bertacchini, 2003) como "un conjunto de posibles conexiones que se establecen entre los usuarios de un espacio intangible pero completamente real en términos de eficiencia productiva y cultural y donde, más que una tradicional relación de vecindad, se tratará de asegurar una conexión efectiva entre los sistemas".

Dentro de la esfera del Derecho inmaterial, el surgimiento de territorios virtuales y digitales presupone una innovación que a veces es difícil de entender; es por ello que algunos autores de la territorialidad del Derecho en la era digital siguen exigiendo un aspecto territorial y físico, sin embargo, la realidad ha demostrado la existencia de un espacio virtual alimentado por las empresas proveedoras del servicio y sus clientes, aun cuando la relación entre estos dos y el territorio, no sea física, por lo que se considera como revolucionaria (BERGÉ, 2016).

Ahora bien, la discusión del Geo-bloqueo gira entorno a la localización geográfica de un cliente. De esta manera, los operadores económicos utilizan tecnologías de Geolocalización (TDG), que se definen como "todas las técnicas y dispositivos que permiten adquirir y difundir información de carácter geográfico" (QUESNOT, 2019). En general, el término Geolocalización se refiere a la acción de recopilar la mayor cantidad de datos de un consumidor y analizar los aspectos de identificación en un mercado.

La dirección IP "es una dirección digital que utilizan las computadoras para comunicarse a través del Protocolo de Internet (IP) " (Escoffier, 2009). La CNIL en Europa en su informe del 20 de junio de 2007 indicó que las direcciones IP son consideradas como datos de carácter personal (Ley Francesa, 1978) y el Tribunal de Justicia de la Unión Europea ha establecido que "esta dirección es un dato protegido de carácter personal, porque permiten la identificación precisa de dichos usuarios" (Sentencia n. C-70, 2010), es decir, cuando su información se utiliza para su geolocalización.

La geolocalización de una persona física puede llevarse a cabo mediante varios procesos que se utilizan con el propósito de ubicar a una persona en el exterior (outdoor) mediante métodos operativos de sistemas satelitales como el GPS (Global Positioning System); GLONASS (Global Navigation Satellite System) y próximamente el sistema de posicionamiento (Galileo); de explotación de redes de comunicaciones terrestres tales como GSM (Sistema global para comunicaciones móviles), UMTS (Sistema universal de telecomunicaciones móviles), LORAN (Navegación de largo alcance), TNT; 
o por el contrario, una ubicación a través de tecnologías (indoor), también llamadas micro-ubicación como WIFI, Bluetooth, Zigbee, Infrarrojos, Ultra sonido, ULB etc.

\subsection{Los mecanismos para evitar el Geo-bloqueo}

Teniendo en cuenta que Geo-bloqueo afecta el derecho al anonimato al utilizar datos personales como la ubicación de un cliente, es indispensable que, dentro de su estudio, se haga referencia a los mecanismos que permiten ocultar la dirección IP y eludir el geo-bloqueo como los Proxies, las redes privadas virtuales (VPN) y el proyecto Tor. entre otros. Los Proxies o servidor proxy "es una computadora de terceros a través de la cual accede a un servicio de Internet, funciona como una especie de búfer entre una computadora y un sitio web" (Orange Developer, 2018). Los dos tipos más populares de proxies son proxies web y proxies HTTP.

Paralelamente, la VPN (Red Privada Virtual) consiste en "un túnel lógico establecido entre dos entidades de una misma red, que permite crear la invisibilidad desde el exterior de los datos que circulan por ella" (Utersinger, 2014), existen VPN gratuitas, pero no son consideradas como seguras, las empresas suelen utilizar redes privadas para permanecer en el anonimato. Finalmente, el software Tor o "The Onion Router" es un navegador conocido por ser un proyecto creado por Estados Unidos con el objetivo de tener una red completamente anónima, es utilizado por otros países para el espionaje militar, Tor es una herramienta anti-censura que permite que "en lugar de establecer la conexión directamente con el sitio a visitar, pasará por varios ordenadores diferentes" (Utersinger, 2014), y permite eludir el Geo-bloqueo. En consecuencia, ante la discriminación geográfica, el usuario de Internet como consumidor se verá obligado a ocultar sus datos de ubicación para poder acceder a bienes y servicios sin ser detectado.

\subsection{La estrategia de un mercado único digital europeo}

Debido a la globalización, la expansión de los mercados, el nacimiento de las nuevas tecnologías y los grandes inventos informáticos, la Unión Europea ha ampliado su política de mercado interior hacia el ámbito digital mediante la estrategia de "mercado único digital" o "The Digital Single Market ("DSM"), la cual fue comunicada el 6 de mayo de 2015 y adoptada el 19 de mayo de 2015, mediante la cual se dispuso la adaptación de las libertades de circulación para los nuevos desafíos que suponen las nuevas tecnologías en el comercio electrónico. Gracias a este mercado único, los nacionales de un país de la Unión Europea no encontrarán fronteras para realizar sus transacciones con otro Estado miembro. Sin embargo, el Geo-bloqueo constituye un obstáculo porque construye barreras virtuales entre los consumidores y fragmenta el mercado.

El desarrollo de una estrategia digital tiene sus raíces en el concepto de economía digital, según el cual la economía de un país debe adaptarse al 
desarrollo tecnológico y de las tecnologías de la informática y las comunicaciones con el objetivo de crear sociedades más modernas e innovadoras. Hoy en día, el desarrollo de un mercado único digital es uno de los objetivos de la estrategia 2020 de la Comisión de la Unión Europea denominada "estrategia para un crecimiento inteligente, sostenible e inclusivo" (commission Européenne, 2020).

2.3 La estrategia del mercado único digital en América latina (MUD) El Banco de Desarrollo de América Latina ((CAF), 2016) propuso el proyecto de estrategia para la creación de un mercado único digital (MUD) en América Latina, dentro del cual se pueden destacar:

- Latinoamérica no cuenta una reglamentación en materia de portabilidad transfronteriza de contenidos en línea.

- En materia de Derechos de autor, los proveedores de servicios en línea envían licencias a cada país, de manera diferente y en algunos casos en ausencia de legislación aplicable debido a que las cláusulas de exclusividad, de no competencia o de cesión de derechos de autor se limitan a la relación contractual entre las partes (presencia de abuso al consumidor), al mismo tiempo, Mercosur no cuenta con ninguna normativa incluyente en materia de derechos de autor, con la excepción de Chile con una reforma reciente de la ley de propiedad intelectual.

- Teniendo en cuenta la falta de recursos y medios digitales de los países latinoamericanos, la problemática del Geo-bloqueo aún no constituye una preocupación prioritaria en la vida de los consumidores latinoamericanos, sin embargo, debido al aumento de uso de contenido digital como consecuencia de las medidas de confinamiento derivadas de la crisis sanitaria COVID-19, se ha evidenciado que las restricciones de acceso a bienes y servicios se convierten en un tema de mayor relevancia para los cyberconsumidores de estos países.

- La práctica de Geo-bloqueo es ampliamente utilizada por las plataformas de Internet en estos países, "en Argentina, Brasil, Chile, Colombia, México y Perú, las principales plataformas dirigen al usuario a contenidos locales basados en su dirección IP; no es posible el acceso a instalaciones extranjeras, excepto Apple y PlayStation, que permiten el acceso a su contenido según el país de origen de la tarjeta de crédito y / o el país donde el usuario creó la cuenta" (Cullen, I. CAF, 2016)".

Finalmente, la estrategia de mercado único digital (MUD) de América Latina ha sido incluida en los objetivos de la "agenda digital 2020" de la CEPAL y actualmente constituye uno de los desafíos del plan de desarrollo digital latinoamericano, confirmado por varios proyectos como el Acuerdo de Cooperación Económica Transpacífica (TPP) y el Foro de Cooperación AsiaPacífico (APEC), la Alianza del Pacífico que están trabajando especialmente 
en telecomunicaciones y comercio electrónico. Igualmente, nuevos proyectos son objeto de desarrollo del mercado digital de América Latina como el proyecto Mesoamérica, el Sistema de Integración Centroamericana y Comunidad del Caribe (CARICOM) y la Comisión Interamericana de Telecomunicaciones de la Organización de Estados americanos (OEA).

\section{La reglamentación para la prohibición del Geo-bloqueo injustificado en Europa}

En el marco de la misión de la Unión Europea para eliminar los obstáculos del mercado digital, el Parlamento Europeo y el Consejo establecieron el Reglamento 2018/302 para prohibir el bloqueo geográfico sin justificación. Esta disposición permite sancionar situaciones discriminatorias y que violan el artículo 20 de la Directiva 2006/123/UE, proporcionando a los Estados miembros una mayor seguridad jurídica.

En cuanto al Geo-bloqueo, el Reglamento 2018/302 es completamente innovador en comparación con otros países, al tomar la iniciativa de prohibir esta práctica cuando no hay justificación. Esta disposición demuestra el criterio restrictivo de la Unión Europea que busca proteger a los consumidores con el objetivo de construir un mercado único digital sólido.

\subsection{Campo de aplicación del reglamento 2018-302}

a. El Reglamento no debe aplicarse a las situaciones puramente internas de un Estado miembro.

b. Para su aplicación se debe tener en cuenta los criterios de la nacionalidad, el lugar de residencia o el lugar de establecimiento del cliente o del comerciante, el lugar de ejecución, los medios de pago utilizados en la transacción o la oferta y la utilización de una interfaz en línea (Reglamento (UE), 2018).

c. Se excluyen del ámbito de aplicación del Reglamento 2018/302 los servicios audiovisuales, específicamente los servicios cuyo objetivo principal sea proporcionar acceso a transmisiones deportivas y que se prestan sobre la base de licencias territoriales exclusivas. Por lo tanto, también debe excluirse el acceso a servicios financieros al por menor, incluidos servicios de pago (Reglamento (UE),2018).

d. Igualmente, los servicios de transporte como las ventas de billetes para el transporte de viajeros quedan excluidos ${ }^{3}$

e. Las condiciones que se negocian individualmente entre el comerciante y los clientes no deben considerarse condiciones generales de acceso a efectos del Reglamento 2018-302.

f. La protección del Reglamento se aplica siempre y cuando su actúe como cliente existiendo una intención de compra por lo que esa protección no debe hacerse extensiva a los clientes que adquieran un

\footnotetext{
${ }^{3}$ Que se detallan en los reglamentos (CE) n.o 1008/2008 (1), (UE) n.o 1177/2010 (2) y (UE) n.o 181/2011 (3) del Parlamento y del Consejo Europeo.
} 
producto o un servicio para la reventa, transformación, procesamiento, alquiler o subcontratación posteriores (Reglamento (UE), 2018).

\subsection{Las principales modificaciones del Reglamento al mercado digital}

a. Del acceso a interfaces en línea

Con relación al acceso a un interfaz en línea, plataforma digital o sitio web, la prohibición del Geo-bloqueo no es absoluta cuando existe un motivo que justifica la restricción de acceso, como por ejemplo la seguridad pública. sin embargo, la reglamentación prevé dos acciones que todo proveedor de servicios o comerciante deben realizar para poder restringir el acceso de un cliente:

En primer lugar, el comerciante debe indicar en el idioma del consumidor una explicación expresa de las razones para restringir su acceso al contenido o servicio en línea, teniendo en cuenta que su justificación debe ser objetiva. En segundo lugar, el consumidor puede aceptar ser redirigido a otra plataforma o sitio web diferente, dando su consentimiento expreso.

b. Del acceso a las ventas en línea

En el ámbito del comercio electrónico, la prohibición de Geo-bloqueo responde al principio de libre circulación de bienes y servicios del mercado interior europeo, en este sentido, el reglamento 2018-302 prevé que cuando un consumidor desee adquirir un producto o servicio online, estará prohibido exigir condiciones de venta diferentes debido a la ubicación geográfica del cliente sin justificación, sin desconocer que los comerciantes son libres de establecer condiciones de venta diferentes siempre que no existan motivos discriminatorios.

Con relación a las condiciones de entrega de productos, los comerciantes podrán imponer restricciones en virtud del principio del libre comercio electrónico sin estar obligados a garantizar plenamente el acceso a los bienes y servicios (Reglamento (UE), 2018).

Por último, el reglamento 2018-302 no podrá contradecir a las restricciones y limitaciones de venta de bienes y servicios impuestas por la legislación del Derecho europeo en materia de comercio electrónico.

c. No discriminación por motivos relacionados con los medios de pago En último lugar, la prohibición de Geo-bloqueo injustificado concierne también las condiciones de pago impuestas a los clientes, prohibiendo todo tipo de discriminación en razón al domicilio bancario y al lugar de pago de los bienes y servicios en otro estado miembro de la Unión Europea. Por lo que, el reglamento 2018-302 prohíbe la imposición de un medio de pago al cliente sin justificación y le da la posibilidad de elegir cual es la oferta de bienes y servicios y la forma de pago que más le conviene. 


\subsection{Eficacidad y sanciones del dispositivo}

Como primera medida, el Reglamento 302/2018 establece que los Estados miembros deben designar uno o más organismos dotados de competencias para supervisar la aplicación de la presente disposición. Al mismo tiempo, deberán determinar si sus órganos serán autoridades judiciales o administrativas y si cuentan con las facultades necesarias para asegurar el cumplimiento de este mecanismo.

Finalmente, los Estados miembros tienen el deber de monitorear las medidas implementadas para sus organismos en caso de una práctica de bloqueo geográfico que se encuentre dentro de su alcance.

Así mismo, este dispositivo prevé medidas que pueden afectar a las condiciones de competencia en un mercado ${ }^{4}$. En este orden de ideas, la Comisión Europea mediante decisión de 17 de diciembre de 2018, sancionó a la empresa Guess con una multa de casi 40 millones de euros por prácticas de Geo-bloqueo en el marco de unos contratos de distribución (Comisión Europea, 2018).

La Comisión Europea en la investigación llevada a cabo en 2017 sobre la empresa Guess había determinado la existencia de acuerdos de distribución en los que la empresa imponía restricciones a los minoristas del uso de marcas en la publicidad de búsqueda online, la negativa sin justificación de una autorización para vender sus productos de marca en línea, y la implementación de limitaciones de acceso a consumidores ubicados en otro territorio que difieren de la del distribuidor, entre otros. En la sentencia, la Comisión Europea consideró que las cláusulas del contrato de distribución establecidas por la empresa Guess constituyen restricciones verticales territoriales en el sentido del artículo 4 del Reglamento 330/2020 y una violación al artículo 110 del TFUE.

En este mismo sentido, en 2017 la Comisión Europea abrió una investigación sobre la empresa de videojuegos Valve, debido a los acuerdos aparentemente realizados con las empresas editoras de videojuegos Bandai Namco, Capcom, Focus Home, Koch Media y ZaniMax en los que impuso "claves de activación" para evitar las ventas transfronterizas a los consumidores, con técnicas de bloqueo geográfico (Comisión Europea, 2019).

\section{Conclusión: Una futura reglamentación del Geo-bloqueo en América latina}

La existencia de un territorio digital ha sido una de las principales razones del crecimiento de las nuevas tecnologías y el desarrollo de métodos para cruzar sus límites y cambiar el juego a favor de las grandes empresas que ofrecen servicios digitales, en este sentido, se evidencia que el Geo-bloqueo ha dejado

\footnotetext{
${ }^{4}$ Ver el articulo 4 parágrafo 2 del reglamento 302/2018 el cual es relativo a los acuerdos de venta de pasivos.
} 
su identidad como medida de protección de los Estados para convertirse en un mecanismo real para limitar a los consumidores y fragmentar un mercado sin razón alguna.

Como se ha demostrado en este estudio, el uso del Geo-bloqueo puede generar efectos negativos contra los consumidores impidiendo el desarrollo justo e igualitario del comercio electrónico, además de constituir un límite a una estrategia del mercado único digital. Sin embargo, la era digital no conoce fronteras y el Geo-bloqueo podría afectar a cualquier consumidor sin importar el territorio en el que se encuentre, siendo América Latina uno de los territorios más afectados de bloqueo geográfico por empresas y plataformas digitales a las que no se tienen acceso y en detrimento de la libertad del consumidor.

Partiendo de este planteamiento final y en procura del desarrollo de un proyecto de creación de mercado único digital para Latinoamérica, se evidencia una necesidad de análisis y estudios doctrinales para adaptar el sistema jurídico de los países de la región para superar los obstáculos presentes en el territorio digital.

En este sentido y con base a la legislación actual europeo se presentan las siguientes recomendaciones ante la práctica injustificada del Geo-bloqueo y una futura reglamentación en América latina:

La utilización de mecanismos para evitar el Geo-bloqueo como primera medida para el cambio. Si bien es cierto, los países latinoamericanos no cuentan con un mercado único digital, la realidad demuestra que todos los países constituyen un mercado digital en plena interacción con cualquier sitio en línea sin importar la nacionalidad del internauta, por ello frente a la falta de regulación en la materia, los consumidores Colombianos se ven en la obligación de acudir a mecanismos tecnológicos para evitar el Geo-bloqueo y ocultar la ubicación, sin embargo, muchos de ellos no proporcionan una seguridad para el navegador y en ocasiones son muy costosos.

Una reglamentación del Geo-bloqueo injustificado que proteja el derecho al anonimato en internet. Tal como quedó establecido, esta práctica puede ser realizada por los países como medida de protección y por los particulares siempre que medie una justificación objetiva, por ello, la prohibición de esta práctica en Europa se limita al Geo-bloqueo como forma de discriminación sin justificación que la acredite, a partir de esta premisa, se concluye que un cierto grado de Geo-bloqueo deberá ser permitido para la protección de los consumidores.

En materia de derecho de autor, el dispositivo de prohibición del Geobloqueo en Europa excluye los contenidos audiovisuales para los que se requiere una licencia territorial. Tal es el caso de la cotidianidad en 
América latina, sin embargo, una reglamentación al respecto deberá luchar contra el abuso de la posición dominante y al derecho del consumidor por parte de las cláusulas abusivas incluidas en los contratos por parte de las grandes plataformas de internet. Resaltando que la reglamentación europea no desconoce la legislación en materia de derechos de autor, lo cual, deberá ser también respetado en América latina.

La línea entre las prácticas anticompetitivas y el bloqueo geográfico se vuelve cada vez más estrecha. Como ha sido analizado, el Geo-bloqueo se considera como una práctica restrictiva de la competencia afectando las condiciones de competencia de un mercado porque ayuda a limitar, cerrar y bloquear a los clientes afectando a la competencia de manera desleal, e impartiendo condiciones diferentes en razón a la ubicación del cliente, es por ello, que una reglamentación resulta primordial.

El desarrollo del derecho digital e inmaterial permitirá una mayor protección del consumidor digital y del avance y estructuración de un mercado digital. Para este punto, Colombia podría ser uno de los países pioneros en la construcción de una reglamentación contra las problemáticas jurídicas de la era económica digital, para ello podría tomarse como referencia la reglamentación europea pues los territorios digitales no conocen fronteras y para su estructuración no es indispensable que exista un mercado único digital en el territorio latinoamericano, más sin embargo, una primera reglamentación ayudara a afrontar los cambios jurídicos actuales con mayor seguridad.

La estrategia de creación de un mercado único digital en Latinoamérica es un proyecto no muy lejano. Los países deberán estar conectados digitalmente y cada análisis o estudio que permita la construcción de este mercado es de vital importancia para poder estar a la altura de los mercados internacionales y adaptar los sistemas jurídicos de los países haciéndolos más competitivos.

De esta manera, Colombia y los países latinoamericanos deberán prepararse para afrontar las problemáticas actuales digitales, para lograr una mejor adaptación del sistema jurídico a la era de la economía digital. Así mismo, la reglamentación europea en este ámbito constituirá un antecedente importante para la aplicación y la lucha contra los efectos del Geo-boqueo. La realidad indica que la revolución digital debe ser utilizada en beneficio del desarrollo siendo importante conocer las doctrinas jurídicas internacionales que permitan facilitar su adaptación para los mercados menos avanzados porque como dijo Jean Claude Juncker, "estoy convencido de que debemos aprovechar mucho mejor las enormes posibilidades que ofrecen las tecnologías digitales que no conocen fronteras". 


\section{Bibliografía}

Bergé, J-S. (2016). La sphère des données et le droit: nouvel espace, nouveaux rapports aux territoires. Journal du droit international, 1153-1173.

Bertacchini, Y. (2003). Territoire physique/Territoire virtuel: quelle cohabitation. Journal of Information Sciences for Decision Making (9), 6.

Comision Europea. (2018). Relativa a un procedimiento en virtud del artículo 101 del Tratado de Funcionamiento de la Unión Europea y del artículo 53 del Acuerdo sobre el Espacio Económico Europeo (Asunto AT. 40428) C/2018/8455

Commission Européenne (2020) "une stratégie pour une croissance intelligente, durable et inclusive" Communication de la commission Européenne 2020

Commission Européenne. (18 mars 2016). Synthèse des réponses à la consultation publique de la Commission européenne réalisée en 2015 sur le géoblocage et d'autres restrictions géographiques appliquées aux achats et à l'accès à l'information dans l'UE. Rapport de synthèse, 11.

Commission Européenne. (5 de abril de 2019) « Antitrust: Commission sends Statements of Objections to Valve and five videogame publishers on "geoblocking" of PC video games Brussels », Comunicado de prensa de la Comusion Europea.

Cullen, I. (2016). Hacia la estrategia para el mercado único digital de América Latina.

Derieux. (2010). Entre esprit libertaire et nécessaire réglementation. A propos de la 'neutralité de l'internet, un atout pour le développement de l'économie numérique. RLDI/ 2010/64(2101), 6-10.

Escoffier, A. (2009). La vie privée à l'heure des mémoires numériques. rapport d'information $\mathrm{n}^{\circ} 441$.

European Commission, E. (2016). Geo-blocking practices in e-commerce - Issues paper presenting initial findings of the e-commerce sector inquiry conducted by the Directorate-General for Competition, Commission Staff Working Document. Brussels: SWD.

Europeo, P. (2013). Discriminacion de consumidores en el Mercado unico digital (Digital Single Market). IP/A/IMCO/ST/2013-03.

Maire, G. (2019). l'accès à Internet, analyse juridique d'une mutation du droit. Revue Lamy droit de l'immatériel (158), 33.

Orange Developer (2018) Généralités sur les VPNs. Recuperado de: https://developer.orange.com/wp-content/uploads/Generalites-sur-les-VPNs.pdf 
Pablo, M. (2017). La transparence du lobbying et l'efficacité du processus décisionnel européen. Faculté des sciences économiques, sociales, politiques et de communication, Université catholique de Louvain, 59.

Parlamento Europeo (7 de diciembre de 2000) Carta de los Derechos Fundamentales: Niza, Francia.

Parlamento Europeo. (12 de diciembre de 2006). Relativa a los servicios en el mercado interior. Directiva 2006/123/CE del Parlamento Europeo.

Parlamento Frances. (6 de enero de 1978) Ley Francesa de la informática y las libertades de la comunicación.

Quesnot, T. e. (2019). Explorer les mobilités partagées à l'ère des Technologies de Géolocalisation. 2.

Reglamento (UE). (25 de noviembre de 2015). Por el que se establecen medidas relativas al acceso a una Internet abierta y se modifican la Directiva 2002/22 / CE y el Reglamento (UE) no 531/2012. Reglamento 2015/2120.

Reglamento (UE). (28 de febrero de 2018). Parlamento Europeo y el Consejo relativo a la prohibición del geo-bloqueo injustificado y otras formas de discriminación. Reglamento 2018/302.

Squire Patton Boggs (2017). Loi numérique: neutralité de l'internet. Revista droit du numerique

Utersinger, M. (2014). Anonymat sur Internet: Protéger sa vie privée. Editions Eyrolles, 144. 